University of Nebraska - Lincoln

DigitalCommons@University of Nebraska - Lincoln

January 2005

\title{
Domain structure and polarization reversal in ferroelectrics studied by atomic force microscopy
}

\author{
Alexei Gruverman \\ University of Nebraska-Lincoln, agruverman2@unl.edu \\ O. Kolosov \\ Joint Research Center for Atom Technology (JRCAT), Angstrom Technology Partnership, 1-1-4 Higashi, \\ Tsukuba, Ibaraki 305, Japan \\ J. Hatano \\ Science University of Tokyo, Noda,Japan \\ K. Takahashi \\ National Institute for Research in Inorganic Materials, 1-1 Namiki, Tsukuba, Ibaraki 305, Japan \\ H. Tokumoto \\ JRCAT, National Institute for Advanced Interdisciplinary Research, 1-1-4 Higashi, Tsukuba, Ibaraki 305, \\ Japan
}

Follow this and additional works at: https://digitalcommons.unl.edu/physicsgruverman

Part of the Physics Commons

Gruverman, Alexei; Kolosov, O.; Hatano, J.; Takahashi, K.; and Tokumoto, H., "Domain structure and polarization reversal in ferroelectrics studied by atomic force microscopy" (2005). Alexei Gruverman Publications. 35.

https://digitalcommons.unl.edu/physicsgruverman/35

This Article is brought to you for free and open access by the Research Papers in Physics and Astronomy at DigitalCommons@University of Nebraska - Lincoln. It has been accepted for inclusion in Alexei Gruverman Publications by an authorized administrator of DigitalCommons@University of Nebraska - Lincoln. 


\title{
Domain structure and polarization reversal in ferroelectrics studied by atomic force microscopy
}

\author{
A. Gruverman a) \\ National Institute for Research in Inorganic Materials, 1-1 Namiki, Tsukuba, Ibaraki 305, Japan \\ O. Kolosov ${ }^{\text {b) }}$ \\ Joint Research Center for Atom Technology (JRCAT), Angstrom Technology Partnership, 1-1-4 Higashi, \\ Tsukuba, Ibaraki 305, Japan \\ J. Hatano \\ Department of Materials Science and Technology, Science University of Tokyo, Noda, Chiba 278, Japan \\ K. Takahashi \\ National Institute for Research in Inorganic Materials, 1-1 Namiki, Tsukuba, Ibaraki 305, Japan \\ H. Tokumoto \\ JRCAT, National Institute for Advanced Interdisciplinary Research, 1-1-4 Higashi, Tsukuba, \\ Ibaraki 305, Japan
}

(Received 11 October 1994; accepted 21 November 1994)

\begin{abstract}
The ferroelectric domain structure and its dynamics under applied electric field have been studied with nanoscale resolution by atomic force microscopy (AFM). Two mechanisms responsible for the contrast between opposite domains are proposed: large built-in domains are delineated in friction mode due to the tip-sample electrostatic interaction, and small domains created by an external field are imaged in topography mode due to piezoelectric deformation of the crystal. The ability of effective control of ferroelectric domains by applying a voltage between the AFM tip and the bottom electrode is demonstrated. It is experimentally confirmed that the sidewise growth of domain proceeds through the nucleation process on the domain wall. (C) 1995 American Vacuum Society.
\end{abstract}

\section{INTRODUCTION}

Ferroelectric materials are a special group of polar dielectrics which possess the ability to switch their spontaneous polarization with an external electric field. The opposite ferroelectric domains have piezoelectric, pyroelectric, electro-optic and nonlinear optic constants which are opposite in sign, and this character of the antipolar domains is the basis for many applications. Over the last few years interest in ferroelectrics has essentially increased as a result of recent achievements in the processing of ferroelectric thin films and their wide application in various electronic and optoelectronic devices. Because the arrangements of domain structures have a direct influence on the macroscopic properties of ferroelectric samples, it is of great importance to be able to examine the ferroelectric domain structure on the smallest possible scale. To visualize the domain structures of ferroelectric materials, several methods have been applied, such as polarization microscopy, surface etching, powder decoration, and electron microscopy. ${ }^{1-4}$ Recently the possibility of revealing ferroelectric domains using atomic force microscopy (AFM) in the micron range has been reported. ${ }^{5}$ AFM promises to be a powerful tool for the study of ferroelectric domains providing the nanoscale resolution of domain structure. ${ }^{6,7}$ Due to the very small curvature of the AFM tip, an extremely high electric field can be generated by applica-

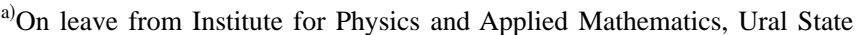
University, Ekaterinburg 620083, Russia.

${ }^{b)}$ On leave from Institute of Chemical Physics, Russian Academy of Sciences, Moscow, Russia. Present address: Department of Materials, University of Oxford, OX1 3PH Oxford, United Kingdom.
}

tion of a comparatively low potential. This makes AFM a very promising technique for the modification of domain structure by local polarization reversal and for the creation of a highly ordered domain structure with an average resolvable spacing close to the physical limit. Another attractive prospect for AFM, not yet realized, would be direct nanoscale imaging of domains during their switching. This would allow one to directly study the nature of ferroelectric behavior.

In the present article, an analysis of the basics of ferroelectric domain contrast during AFM imaging is given. Using a conductive cantilever under variable electric potential, a controllable local switching of polarization in the ferroelectric crystal has been realized and the dynamics of domain nucleation, growth and interaction, have been studied with nanoscale resolution.

\section{EXPERIMENT}

To investigate the domain contrast in AFM we used a guanidinium aluminum sulfate hexahydrate (GASH) crystal, chemical formula $\mathrm{C}\left(\mathrm{NH}_{2}\right)_{3} \mathrm{Al}\left(\mathrm{SO}_{4}\right)_{2} 6 \mathrm{H}_{2} \mathrm{O}$. GASH is an uniaxial ferroelectric, belonging to the trigonal crystal system (space group P $31 \mathrm{~m}$ ). ${ }^{8}$ The spontaneous polarization $P_{s}$ is about $0.35 \mu \mathrm{C} / \mathrm{cm}^{2}$, the dielectric constant, $\epsilon_{c}$ along the polar axis is 15 , and the coercive field is about $1.5 \times 10^{5} \mathrm{~V} / \mathrm{m}$. GASH has a perfect cleavage plane normal to the polar axis $c$, which is suitable for studying the domain structure. The thickness of crystal plates, which were cleaved in air, varied from 50 to $200 \mu \mathrm{m}$. The samples were investigated in an ambient environment by means of a commercially available microscope (SPA 300, Seiko Instruments) operating both in topography and friction modes. Static and dynamic domain 
patterns have been delineated with nanoscale resolution. To distinguish the contrast associated with topography from that related to electrostatic tip charging, we used two types of cantilevers: a standard, with a nonconductive $\mathrm{Si}_{3} \mathrm{~N}_{4}$ tip and a gold coated conductive tip and cantilever. Both cantilevers had a rigidity of $0.09 \mathrm{~N} / \mathrm{m}$ and the tips had a radius of curvature $R$ of about $50 \mathrm{~nm}$. The force between the tip and sample varied from 1 to $3 \mathrm{nN}$. To reverse the polarization, voltage pulses of variable width, amplitude, and polarity were applied between the bottom electrode and the AFM tip. The pulse amplitude ranged from -5 to $5 \mathrm{~V}$ with the pulse width between 1 and $10 \mathrm{~s}$.

In order to verify the domain structure revealed by AFM the GASH samples were studied by a powder deposition technique which is useful for revealing charged surfaces. ${ }^{1}$ The powder patterns were produced by immersing the samples in a diluted developer. The positively charged carbon particles were attracted to the negatively charged ferroelectric domains and covered their entire area. After the sample was rinsed and the developer was washed off, the sample was cleaved perpendicular to its polar axis so as to make it as thin as possible. The cleaved surface was examined by AFM. Since the GASH is a transparent crystal, by using optical microscope it was possible to position the AFM tip exactly on the domain site revealed by powder deposition technique on the opposite surface.

\section{RESULTS AND DISCUSSION}

Figure 1(a) shows the domain pattern delineated by a powder deposition technique. A few domains of reversed polarity whose sizes range from 5 to $80 \mu \mathrm{m}$ may be seen. Figure 1(b) shows the AFM friction image of the opposite surface taken from the central part of Fig. 1(a). Only a large domain revealed by the powder deposition technique is exposed in the AFM image, and the small domains are not found in AFM. This effect is consistent with results obtained by Szczesniak et al. who have found that the dimensions of small circular domains along the polar axis are comparable to those on the polar surface and that small circular domains do not extend through the bulk of the crystal. ${ }^{3}$ The size of a large domain on the AFM image (about $15 \mu \mathrm{m}$ ) is smaller than that found in the powder pattern (about $50 \mu \mathrm{m}$ ), apparently due to the inclination of the domain walls from the polar direction. From the AFM topography image the difference in height between opposite domains was found to be about $1 \mathrm{~nm}$.

The origin of domain contrast observed in the friction mode can be explained by the difference in lateral forces acting on the AFM tip from the domains with different direction of spontaneous polarization. ${ }^{5}$ This mechanism of domain contrast becomes significant during acquisition of the image when the tip is scanning perpendicular to the length axis of the cantilever. Let us assume that some charge could be induced in the silicon nitride tip by polarization charges of the ferroelectric sample. In this case a lateral electrostatic force additionally acting on the tip causes a twisting of the cantilever along its length axis (Fig. 2). Assuming that the relaxation time of the tip is much longer than the scanning time, the induced charge distribution will be the same even
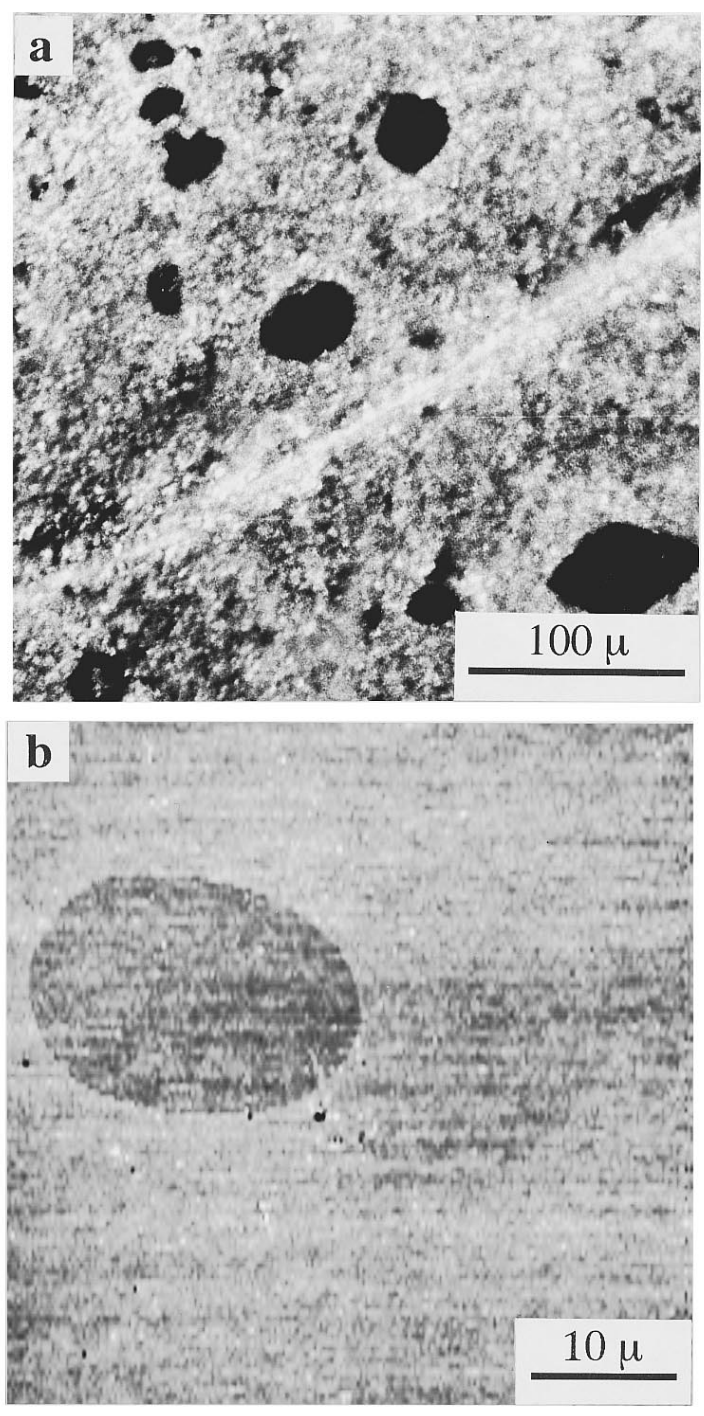

FIG. 1. (a) Microphotograph of a powder pattern on a GASH cleavage surface (the carbon powder covers negatively charged domains). (b) The AFM image of the opposite surface showing the central part of (a) at higher magnification. The AFM was operated in friction mode.

when the tip crosses the domain boundary. Therefore, the sign of the electrostatic interaction force between the tip and sample will be changed, and the resulting change in the lateral force would make it possible to reveal the opposite domains on the cleavage surface. To confirm that electrostatic charges are accounted for the observed domain contrast, the electrically grounded cantilever with conductive tip was used for acquiring the AFM image. No domain contrast was observed, evidently due to the absence of the interaction between the polarization charges and the tip, held under zero potential.

It has been found that an applied voltage leads to a modification of the surface: a voltage of a particular sign created new domains, whereas a potential of opposite sign erased these domains or formed etch holes. A typical etch hole of 6 $\mathrm{nm}$ deep and $50 \mathrm{~nm}$ in diameter created on a freshly cleaved surface by a positive voltage pulse is shown in Fig. 3(a). A matrix of four reversed domains was written by application of negative voltage pulses at the selected points on the sur- 


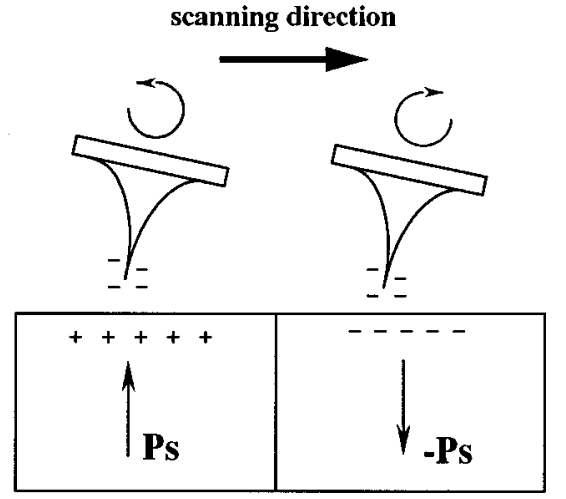

FIG. 2. A model to illustrate the origin of domain contrast in the friction mode. On the left side, the tip scanning the positive domain acquires a negative charge. The lateral component of the attractive electrostatic force leads to a twisting of the cantilever counterclockwise. On the right side, the tip scanning the negative domain retains induced negative charge. The lateral component of repulsive force twists the cantilever clockwise.

face [Fig. 3(b)]. The domains appeared as circular protrusions of $100-150 \mathrm{~nm}$ in diameter and 3-5 nm in height. In principle it was possible to write the domain pattern of any desired configuration with an average resolvable spacing of $200 \mathrm{~nm}$. The pattern in Fig. 3(c) was obtained by scanning with the tip held under a negative dc voltage shortly after the image shown in Fig. 3(b) was taken. As a result the square area containing four domains was completely repolarized.

Figure 4 demonstrates the ability of domain control by the erasing of previously written domains. Stable domains of radius $r$ as small as $100 \mathrm{~nm}$ that were created by the application of negative voltage pulses were subsequently reversed by the AFM tip under positive bias positioned at the domains sites. An additional confirmation that domains were erased due to the local polarization reversal (not just due to the charge deposition) is the fact that no domain contrast was observed in the friction mode after the application of a positive voltage pulse.

To describe quantitatively the domain formation in an applied field, we considered the elementary process of domain nucleation and growth. The electric field, produced by the tip of radius $R$, positioned close to the surface of the object with dielectric permittivity $\epsilon_{a} \gg 1$, can be estimated by its charge $q=4 \pi \epsilon_{a} \epsilon_{0} R V$, acquired due to the applied voltage $V$ (Fig. 4), as

$$
E=q /\left[4 \pi \epsilon_{a} \epsilon_{0}(z+R)^{2}\right]=R V /(z+R)^{2},
$$

where $z$ is the distance from the surface. Therefore, the electric field $E_{S}$, produced by the tip near the surface $(z \ll R)$ due to an applied voltage of $3 \mathrm{~V}$, can be estimated as $E_{s}=V / R=6 \times 10^{7} \mathrm{~V} / \mathrm{m}$. This field is much higher than the coercive field, so one could expect the formation of a domain nucleus of reversed polarization on the sample surface facing the tip. We assume that this nucleus is a half of a prolated spheroid of length $l$ and of radius $r$, aligned in the polar direction. Due to the growth of the reversed domain the energy of the crystal will be changed by an amount ${ }^{9}$

$$
U=-\frac{4}{3} E P_{s} \pi r^{2} l+\frac{1}{2} \pi^{2} r l \sigma+U_{d},
$$

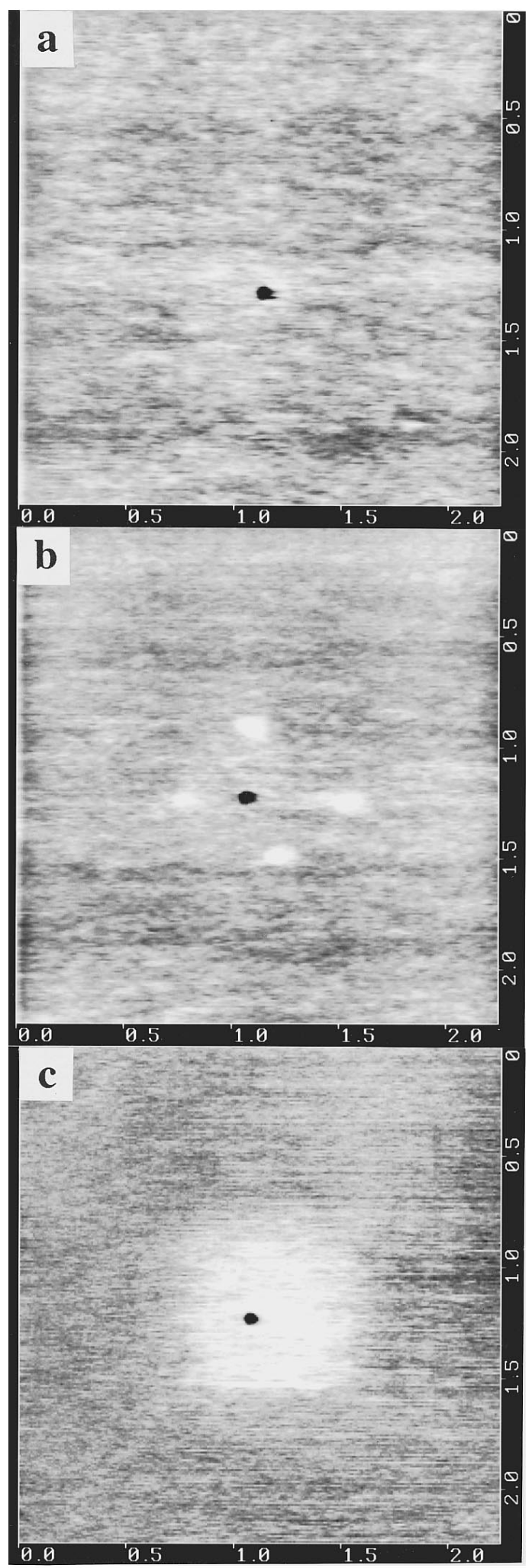

FIG. 3. AFM topography images of a cleavage surface modified by the electric field: (a) an etch hole written by a positive voltage pulse, $V=3 \mathrm{~V}$, duration $2 \mathrm{~s}$; (b) a domain pattern, containing four reversed domains written by negative pulses, $V=-3 \mathrm{~V}$, duration $3 \mathrm{~s}$; (c) square-shape reversed domain obtained by scanning with the tip under a negative dc voltage of $4 \mathrm{~V}$. 

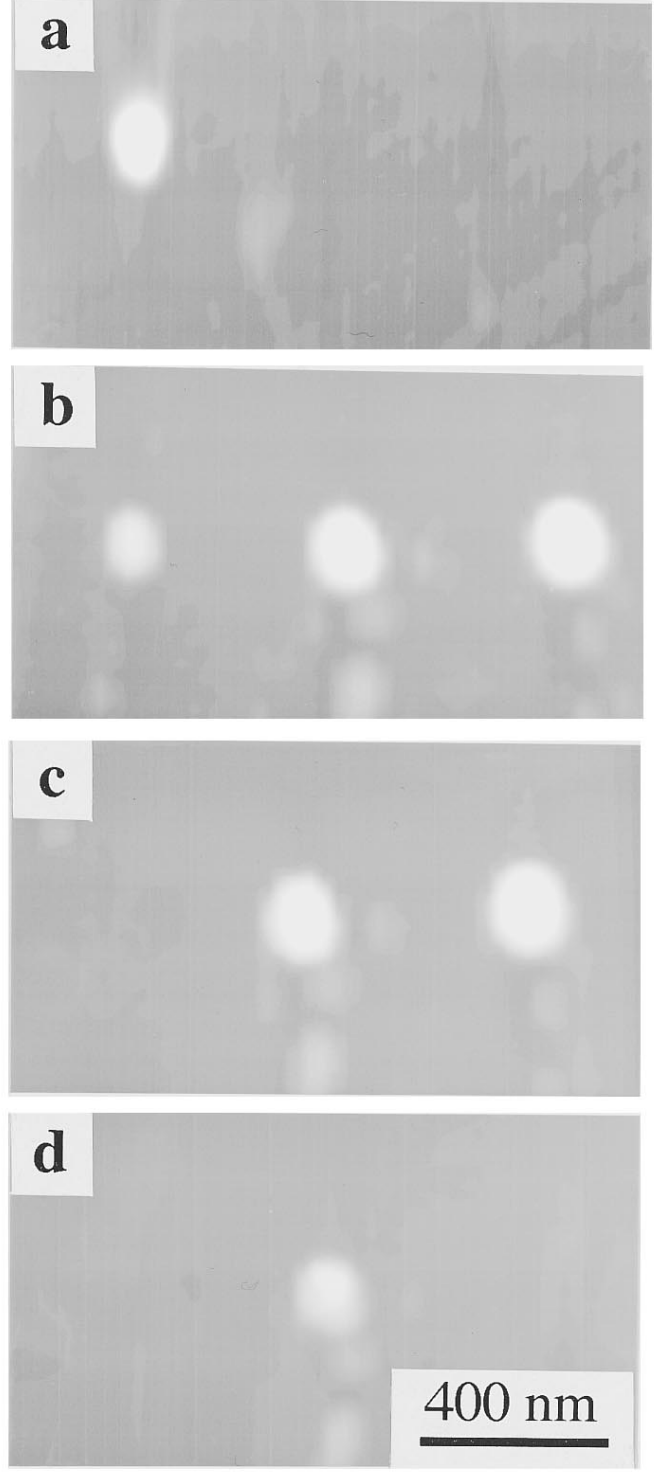

FIG. 4. AFM topography images of domain patterns obtained as a result of domain formation by negative voltage pulses (a), (b) and domain shrinkage under positive voltage pulses (c), (d).

where the expression for the depolarization energy $U_{d}$ was given by Landauer. ${ }^{10}$ The first term in Eq. (2) is associated with the interaction of the applied field with the ions and the second one is the energy required to create a new domain wall. Assuming that $r \ll l$ and using the values $E_{s}=6 \times 10^{7}$ $\mathrm{V} / \mathrm{m}$, and $\sigma=0.3 \times 10^{-3} \mathrm{~J} / \mathrm{m}^{2}{ }^{11}$ it was found that $U$ had its maximum at $r_{c}=15 \mathrm{~nm}$, thereby giving the radius of the critical nucleus.

It is assumed that the contrast of newly formed domains is due to the piezoelectric deformation of the sample. It is well known that the depolarizing field of the ferroelectric crystal is neutralized due to the bulk conductivity of the crystal and by the adsorption of free charges on the polar surfaces. Assuming that there are no free charges in the GASH crystal, the depolarizing field would be compensated only by the adsorbed surface charges. The formation of a domain of reversed polarity will be accompanied by the appearance of a strong uncompensated depolarizing field since there is not

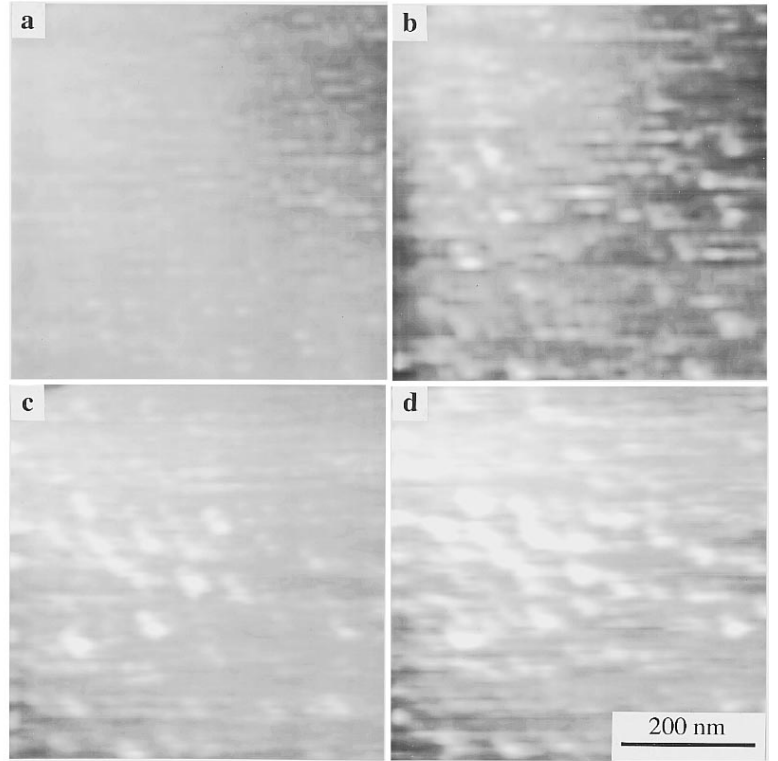

FIG. 5. AFM images illustrating the dynamics of domain growth during continuous scanning under a voltage of $4 \mathrm{~V}$ applied to the tip. Scanning speed was $4 \mathrm{~Hz}$.

enough time to adsorb an appreciable amount of charge on the surface. So after turning off the applied field, the depolarizing field of the new domain gives rise to the piezoelectric strain of the crystal.

There are two parameters involved in the piezoelectric interaction, namely, the depolarizing field $E_{d}$ and the piezoelectric constant. Taking into account only $d_{33}$ constant and assuming that the domain extends through the bulk of the sample, the profile variation $h$ can be approximately estimated as

$$
h=2 d \times E_{d} \times d_{33},
$$

where $d$ is the sample thickness. Since in GASH $d_{33}=2 \times 10^{-12} \mathrm{~m} / \mathrm{V}$ and $E_{d}=2.5 \times 10^{7} \mathrm{~V} / \mathrm{m}^{12}$ for $d=50 \mu \mathrm{m}$ we obtain a value for $h$ of about $5 \mathrm{~nm}$, and this is of the same order as the experimentally measured values. A subsequent adsorption process would deposit compensating charges on the base area of the domain on the polar surface, which should lead to the degradation of topography contrast. Indeed, it was found that the height of the central domain in Fig. 4(d) (about $2.5 \mathrm{~nm}$ ) is smaller than that shown in Fig. 4(c) (about $4 \mathrm{~nm}$ ).

To investigate the dynamics of the domain growth we acquired AFM images while the scanning tip was under potential, generating domain growth. Depending on the applied voltage and scanning speed, the switching of polarization proceeds in different ways. Under scanning with a voltage of $4 \mathrm{~V}$ and scanning speed of $4 \mathrm{~Hz}$ the polarization reversed through the appearance of a number of new circular domains (of diameter 30-50 nm) which became shapeless after coalescence (Fig. 5). No preference in domain wall orientation was observed in this case. Under the lower voltage and slower scanning rate of $1 \mathrm{~Hz}$ the behavior of domain structure during polarization reversal is qualitatively different. In 

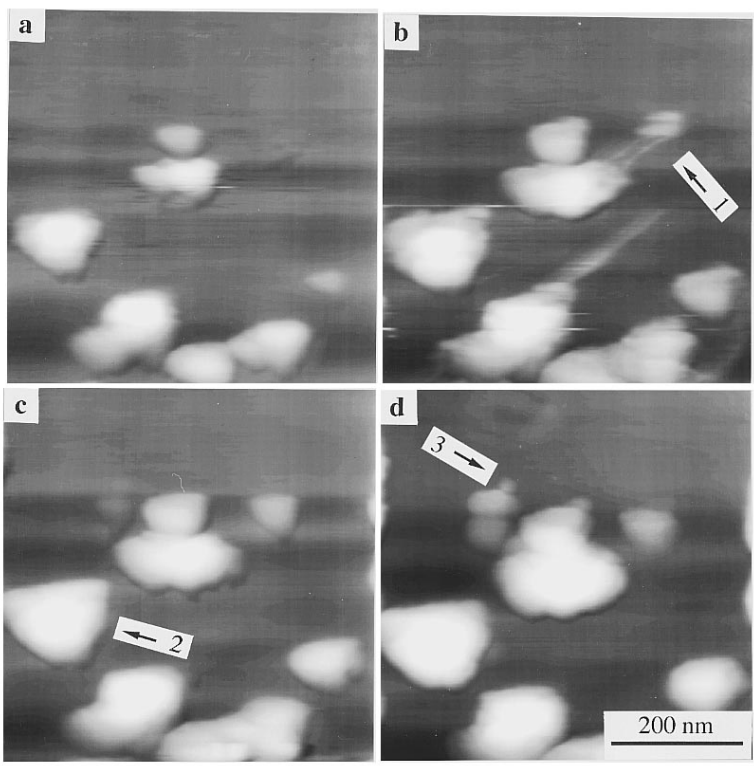

FIG. 6. AFM images illustrating the dynamics of domain growth during continuous scanning under a voltage of $3 \mathrm{~V}$ applied to the tip. Scanning speed was $1 \mathrm{~Hz}$. From the series of subsequent images (a)-(d) it is possible to observe the "remote generation" of the new domain (1), growth of triangular domains (2), and the domain nucleation on the walls (3).

this case, there are fewer domains than in the previous case. As can be seen from the sequence of AFM images in Fig. 6, the polarization is being reversed mainly through the sideways expansion of these domains. After the formation of domains [Fig. 6(a)] their subsequent lateral growth begins [Fig. 6(b)]. It was found that the domains that developed earlier encouraged the formation of new ones by the generation of the nuclei in the direction of crystallographic $a$ axis [Fig. 6(b)] so that new domains appear at some distance from the parent domains [Fig. 6(c)]. The appearance of unusual triangular domains in GASH [Fig. 6(c)] has been reported for the first time, whereas circular or shapeless domains are commonly observed at room temperature. ${ }^{2}$ The walls of triangular domains were perpendicular to the crystallographic $a$ axes of the crystal and were rotated by $30^{\circ}$ with respect to the cleavage steps.

For the first time, the formation of nuclei of reversed polarity on the wall of the domain, growing in the lateral direction was experimentally observed [Fig. 6(d)]. It is commonly accepted that the only physical model which successfully explains the principal experimental results on lateral growth of ferroelectric domains is the nucleation model, analyzed in detail by Miller and Weinreich. ${ }^{9}$ This model assumes that an apparent sideways (lateral) wall motion is caused by the nucleation and subsequent two-dimensional growth of reversed domains on the existing wall. The size of nuclei observed in the present experiment (approximately $30 \mathrm{~nm}$ in diameter) is consistent with our estimations of $r_{c}$. This gives us grounds for considering their formation as an elementary act of domain wall motion and confirming the idea that the motion of the domain wall proceeds through the nucleation process on the wall. ${ }^{9}$

\section{CONCLUSIONS}

The nanoscale visualization and control of the ferroelectric domain structure were reported. The origin of the domain contrast was discussed and the mechanisms of contrast were proposed, based on the piezoelectric deformation of the crystal surface and electrostatic interaction between tip and ferroelectric domains. The dynamics of domain growth was directly observed with a nanoscale resolution. The estimations of nucleus size are consistent with our experimental results, and show that the control of the domains on the nanometer scale can be easily provided by AFM.

\section{ACKNOWLEDGMENTS}

One of the authors (A.G.) would like to thank the Science and Technology Agency of Japan for the provision of a research Fellowship throughout the duration of this study. This work was partially supported by the New Energy and Industrial Technology Development Organization (NEDO), Japan. The support from the Mechanical Engineering Laboratory is highly appreciated.

${ }^{1}$ V. Ya. Shur, A. L. Gruverman, and E. L. Rumyantsev, Ferroelectrics 111, 123 (1990).

${ }^{2}$ J. Hatano, F. Suda, and H. Futama, Jap. J. Appl. Phys. 12, 1644 (1973).

${ }^{3}$ L. Szczesniak, K.-P. Meyer, H. Blumtritt, B. Hilczer, R. Le Bihan, and E.

H. Boudjema, Phys. Status Solidi A 88, 93 (1985).

${ }^{4}$ R. Le Bihan, Ferroelectrics 97, 19 (1989).

${ }^{5}$ R. Luthi, H. Haefke, K.-P. Meyer, E. Meyer, L. Howald, and H.-J. Guntherodt, Surf. Sci. 285, L498 (1993).

${ }^{6}$ G. Binnig, C. F. Quate, and Ch. Gerber, Phys. Rev. Lett. 56, 930 (1986).

${ }^{7}$ D. Sarid and V. Elings, J. Vac. Sci. Technol. B 9, 431 (1991).

${ }^{8}$ A. N. Holden, B. T. Matthias, W. J. Merz, and J. P. Remeika, Phys. Rev. 98, 546 (1955).

${ }^{9}$ R. C. Miller and G. Weinreich, Phys. Rev. 117, 1460 (1960).

${ }^{10}$ R. Landauer, J. Appl. Phys. 28, 227 (1957).

${ }^{11}$ F. Suda, J. Hatano, and H. Futama, J. Phys. Soc. Jpn. 45, 916 (1978).

${ }^{12}$ I. S. Zheludev, Physics of Crystalline Dielectrics (Plenum, New York, 1971). 